\title{
High-Resolution 3-D Refractive Index Tomography and 2-D Synthetic Aperture Imaging of Live Phytoplankton
}

\author{
SangYun Lee ${ }^{1}$, Kyoohyun Kim ${ }^{1}$, Adam Mubarok ${ }^{2}$, Adisetyo Panduwirawan ${ }^{2}$, \\ KyeoReh Lee ${ }^{1}$, Shinhwa Lee ${ }^{3}$, HyunJoo Park ${ }^{1 \dagger}$, and YongKeun Park ${ }^{1 *}$ \\ ${ }^{I}$ Department of Physics, Korea Advanced Institutes of Science and Technology, Daejeon 305-701, Korea \\ ${ }^{2}$ Department of Physics, Bandung Institute of Technology, Bandung, \\ Jalan Ganeca 10, Indonesia \\ ${ }^{3}$ Department of Biological Sciences, Korea Advanced Institutes of Science and Technology, \\ Daejeon 305-701, Korea
}

(Received October 7, 2014 : revised December 4, 2014 : accepted December 5, 2014)

\begin{abstract}
Optical measurements of the morphological and biochemical imaging of phytoplankton are presented. Employing quantitative phase imaging techniques, 3-D refractive index maps and high-resolution 2-D quantitative phase images of individual live phytoplankton are simultaneously obtained without exogenous labeling agents. In addition, biochemical information of individual phytoplankton including volume, mass, and density of individual phytoplankton are also quantitatively obtained from the measured refractive index distributions. We expect the present method to become a powerful tool for the study of phytoplankton.

Keywords : Phytoplankton, Plankton, Quantitative phase imaging, Diffraction optical tomography OCIS codes : (090.0090) Holography; (180.3170) Interference microscopy; (170.3880) Medical and biological imaging
\end{abstract}

\section{INTRODUCTION}

Phytoplankton are major food sources in aquatic ecosystems and play an important role in the global cycle of carbon and silicon, which has led many researchers to focus their efforts on investigating their diverse characteristics [1]. To research this microscopic organism in detail, an appropriate imaging system is essential. Several optical microscopic techniques have been used for the study of phytoplankton $[1,2]$. However, conventional light microscopy techniques have severe drawbacks in imaging and quantitative studying of phytoplankton. For example, bright-field microscopy only produces low imaging contrasts for transparent objects such as phytoplankton. Phase contrast [3] or differential interference contrast (DIC) microscopy [4] provide enhanced imaging contrast for phytoplankton via interference; however, neither the phase contrast nor DIC microscopy provides quantitative information, and they are not capable of 3-D imaging. Although confocal laser scanning microscopy [5] provides the 3-D structures of phytoplankton, samples usually need to be labelled with exogenous agents such as fluorescent proteins or dyes, which cause phototoxicity, photobleaching, and interference with intrinsic cellular metabolism [6].

Recently, there has been an emergence of quantitative phase imaging (QPI) or digital holography techniques and their applications in the study of transparent phase objects such as biological cells and tissues [7-13]. The QPI techniques - based on light interferometry - quantitatively measure optical path length shifts introduced by the refractive index (RI) distribution in a specimen. Several QPI techniques have been extensively employed for investigating phytoplankton research, as summarized in Fig. 1. Using digital in-line holographic microscopy or digital holographic microscopy based on Mach-Zehnder interferometry, the shapes, sizes, and 3-D positions of living organelles were measured [14-22]. However, previous works have only focused on the 2-D imaging of phytoplankton and 3-D internal structures of individual live phytoplankton have not been measured yet, largely owing to the limitation of tomographic capability of conventional in-line holography or digital holographic microscopy. We note a notable exception in which 3-D RI maps of non-living diatom skeletons have been measured

\footnotetext{
*Corresponding authors: *yk.park@kaist.ac.kr, ${ }^{\dagger}$ skdurl20@kaist.ac.kr

Color versions of one or more of the figures in this paper are available online.
} 


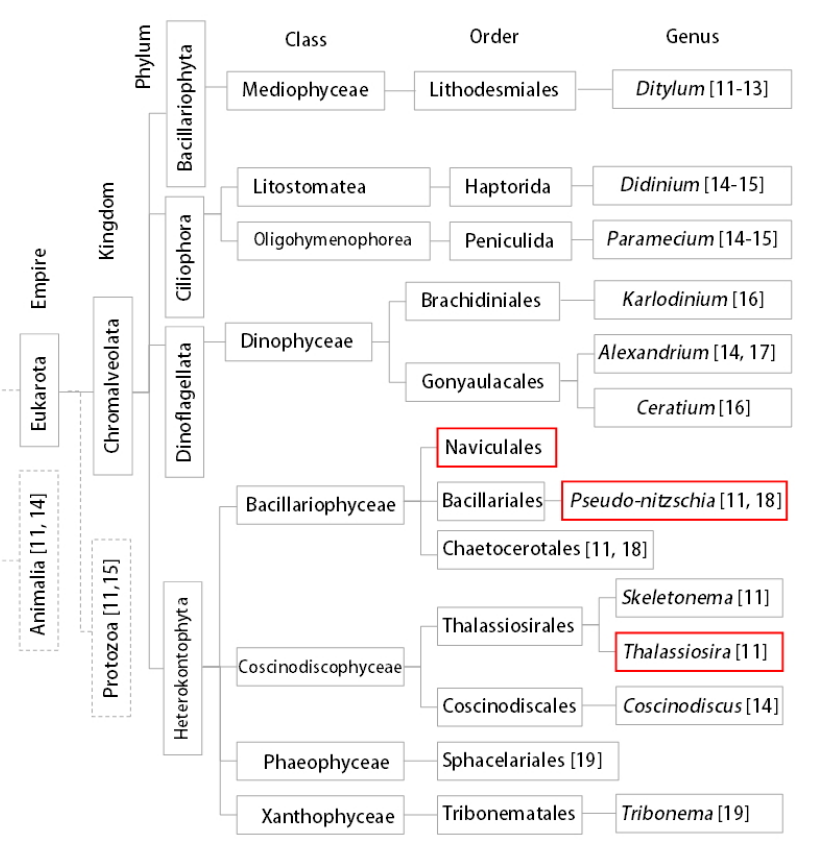

FIG. 1. Classification tree of representative phytoplankton species which have been studied using holographic techniques with references. Red boxes: species measured in the current study.

\section{[23].}

Here we present the measurements of high-resolution 3-D RI maps of phytoplankton employing optical diffraction tomography [24-26]. Multiple 2-D QPI images of individual phytoplankton are recorded with various angles of illumination, from which a 3-D RI map of the sample is reconstructed taking into account the effects of optical diffraction, analogous to computed tomography in X-ray. This measurement of a 3-D RI map provides the quantitative structural and biochemical information of phytoplankton including the dry mass, the volume, and the cytoplasmic density of phytoplankton. Furthermore, the measured set of multiple 2-D QPI images of phytoplankton recorded with various angles of illumination was also utilized for generating a high-resolution 2-D QPI image employing the synthetic aperture algorithm [27], which provides the spatial resolution beyond the diffraction limit determined by the numerical aperture (NA) of a used imaging system. The measured 2-D and 3-D quantitative phase images of phytoplankton and the parameters obtained clearly demonstrate the viability of the present method as a useful tool with unique advantages for the study of phytoplankton.

\section{METHODS}

\subsection{Optical Setup}

To measure the quantitative phase images of individual phytoplankton, we employed quantitative phase microscopy based on Mach-Zehnder interferometry (Fig. 2). The experi-

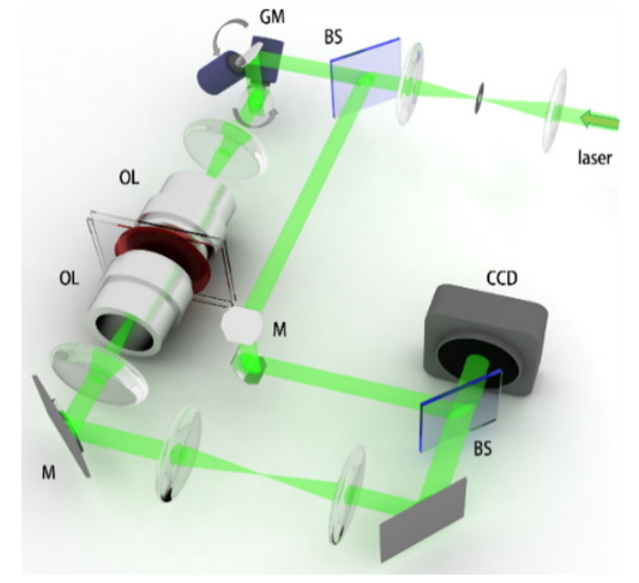

FIG. 2. Experimental optics setup based on Mach-Zehnder interferometry equipped with a two-axis rotating mirror based on galvanometer. BS: beam splitter; GM: galvanometer mirror; OL: objective lens; M: mirror; CCD: camera.

mental setup is equipped with a two-axis galvanometer mirror (GM, GVS012/M, Thorlabs, USA), which varies the illumination angle of an incident beam for the tomographic measurement. This setup is conceptually comparable with setups used in previous works [24, 28].

For an illumination source, a diode-pumped solid state (DPSS) laser ( $\lambda=532 \mathrm{~nm}, 50 \mathrm{~mW}$, Cobolt Co., Solna, Sweden) is used. After spatial filtering, a laser beam is split into two beams by a beam splitter (BS, BS016, Thorlabs, USA). One of the beams serves as a reference beam and the other beam, which traverses the beam splitter and passes through a sample positioned in the stage of an inverted microscope, serves as a sample beam. These two beams are then recombined by another BS in front of the camera with a slightly tilted angle, and resultant off-axis interference patterns are captured by the high-speed CMOS camera (Neo sCMOS, ANDOR Inc., Northern Ireland, UK). One objective lens (UPLFLN, $\times 60, \mathrm{NA}=0.9$, Olympus Inc., San Diego, CA, USA) positioned in front of the sample stage was used as a condenser lens for illuminating purposes. The total magnification of the imaging system is 240 , consisting of the high NA imaging objective lens (PLAPON, $\times 60$, Oil immersion, NA $=1.42$, Olympus Inc., San Diego, CA, USA) and an additional $4-f$ telescopic imaging system. The field of view at the sample plane is $48.1 \times 47.7 \mu^{2}$, which corresponds to $1,776 \times 1,760$ pixels with a pixel size of $6.5 \mu \mathrm{m}$ at the CCD plane.

At the microscopic stage, a sample is illuminated by a plane laser beam, and the angle of illumination can be systematically controlled by rotating the two-axis galvanomirror. For one sample, a set of 300 interferograms with various angles of illumination is recorded at a frame rate of 100 Hz. From the set of measured interferograms (Fig. 3(a)), the corresponding phase and amplitude information (Fig. 3(b) and (c)) of the sample can be retrieved using an appropriate phase retrieval algorithm [29]. These holograms 


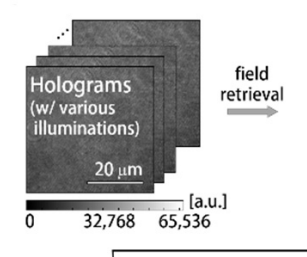

(a)

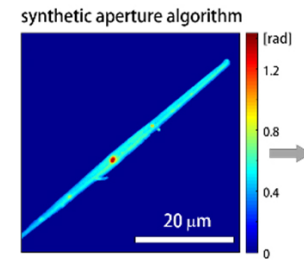

(d)

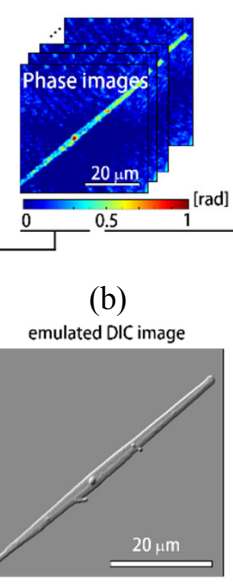

(e)

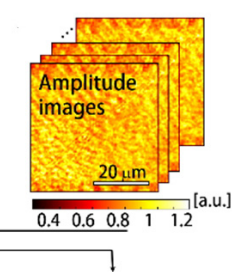

(c)

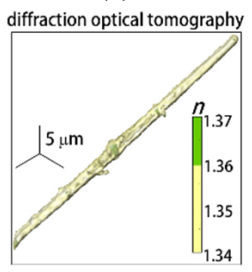

(f)
FIG. 3. A schematic flowchart depicting the process for various QPI imaging modalities. (a) A set of measured holograms of phytoplankton obtained with various illumination angles. A set of (b) phase and (c) amplitude maps retrieved from the holograms in (a) via the optical field retrieval process. (d) 2-D high-resolution phase image obtained with the synthetic aperture algorithm, (e) emulated DIC image calculated from (d). (f) Reconstructed 3-D RI distribution of the phytoplankton.

measured with different illumination angles are then used to reconstruct the high-resolution 2-D quantitative phase map using synthetic aperture algorithm [27] and 3-D RI tomogram image of a sample employing optical diffraction tomography [24-26], which is explained in detail in the following section.

\subsection{Image Reconstruction Processes}

In order to construct 2-D high-resolution phase images of phytoplankton (Fig. 3(d), we employed the synthetic aperture imaging algorithm [27]. The principle of the synthetic aperture algorithm is to numerically extend the aperture of an imaging system in order to achieve high spatial resolution and high signal-to-noise ratio (SNR) [30, 31]. Measured holograms of a sample obtained with various illumination angles contain information on the scattering potential of a sample, which cannot be accessed by a conventional optical imaging system with a fixed aperture. Employing the synthetic aperture algorithm, this information can be fully harnessed and reconstructed in the 2-D Fourier space, and finally provides a high-resolution 2-D phase image of a sample beyond the diffraction-limit determined by NA of the optical imaging system used. The representative phase image obtained with the aperture synthesis algorithm is shown in Fig. 3(d).

Compared to the phase image directly measured from the QPI system (Fig. 3(b), the phase image obtained with the aperture synthesis algorithm (Fig. 3(d)) provides higher spatial resolution and SNR ratio. In conventional quantitative phase images, the maximum spatial frequency $k_{\max }$ which determined the resolving power is determined as $\left|k_{\max }\right|=2$ $\pi N A_{\text {img }} / \lambda$, where $N A_{\text {imag }}$ denotes the $N A$ of the imaging objective lens. Whereas the $k_{\max }$ achieved by the synthetic aperture algorithm is $\left|k_{\max }\right|=2 \pi\left(N A_{\text {illum }}+N A_{\text {img }}\right) / \lambda$. In this case, $N A_{\text {illum }}$ denotes the NA of the condenser objective lens. In addition, using the synthetic aperture imaging, the SNR ratio can be significantly enhanced by reducing speckle noise. Moreover, the emulated DIC image (Fig. 3(e)) can also be numerically obtained. A DIC image can be calculated by interfering a synthetic aperture image, containing both amplitude and phase information, with a laterally translated synthetic aperture image [32]. Internal structures of phytoplankton can be clearly seen in the emulated DIC image, because the imaging contrast of DIC images depends on optical phase gradient.

In order to construct 3-D RI maps of phytoplankton, we employed an optical diffraction tomography algorithm. Based on the Fourier diffraction theorem [33], a set of measured 2-D optical fields can be mapped onto a 3-D scattering potential spectrum. The spatial frequency information of a 2-D optical field measured with a plane wave with a specific angle of illumination corresponds to a hemispheric area, so called Ewald's surface in 3-D Fourier space. Due to the limited NA of an objective lens, some information is inevitably inaccessible in the 3-D Fourier space. This missing information can be filled using an iteration method with appropriate assumptions [34]. Finally, the 3-D RI distribution of a sample can be converted from the 3-D scattering potential spectrum via 3-D inverse Fourier transformation. The representative 3-D RI map of phytoplankton is presented in Fig. 3(f), which shows high-resolution structural information of phytoplankton in 3-D with the quantitative RI values. The details on optical diffraction tomography and the custom-made MatLab codes used, which can be found elsewhere [26].

\section{RESULTS}

\subsection{Quantitative Holographic Images of Individual Phytoplankton}

To demonstrate the capability of quantitative imaging of phytoplankton, we measured 4, 11, 12 of spherical-, rodshaped-, and non-spherical phytoplankton, respectively. These phytoplankton samples were collected from a pond in Daejeon, South Korea. The $50 \mu \mathrm{L}$ of pond water was directly loaded between two coverslips $(24 \times 50 \mathrm{~mm}$, Matsunami, Japan) without adding any exogenous agents. The holographic images of representative individual phytoplankton are depicted in Fig. 3. The high-resolution 2-D phase images obtained with the synthetic aperture algorithm (Figs. 4(a), (d), and (g)], the emulated DIC (Figs. 4(b), (e), and (h)) and the 3-D RI distributions (Figs. 4(c), (f), and (i)) of individual spherical-, rod-shaped-, and non-spherical phytoplankton were reconstructed from sets of measured 2-D phase images, respectively (See Methods).

Based on the measured morphology and internal structures, 
the measured spherical phytoplankton (Fig. 4(a)-(c)) seem to belong to Thalassiosira decipiens. Overall morphology and the internal structures with high RI [indicated with the arrows in Figs. 4(a)-(b)] were seen in both the high-resolution 2-D phase and DIC images. The 3-D RI distribution of the spherical phytoplankton (Fig. 4(c)) clearly reveals the complex internal structures of the phytoplankton. The nucleus has higher RI value $(n>1.36)$ than cytoplasm [the area with green color in Fig. 4(c)]. Furthermore, the retrieved values of RI can serves as intrinsic imaging contrast for phytoplankton, which can be utilized for the extraction of various quantitative imaging parameters of phytoplankton including the mean RI $\langle n\rangle$, dry mass $M$, and the volume $V$ of individual phytoplankton. The calculated quantitative parameters of the spherical phytoplankton are indicated in Fig. 4(c). The rod-shaped phytoplankton (Figs. 4(d)-(f)) seem to belong to Pseudo-nitzschia australis based on its distinctive rod-like shape and the morphology of internal structure examined from the measured 2-D phase image and 3-D RI map. The nucleus structure with high optical thickness is clearly seen in the middle of the rod-shape phytoplankton in both the high-resolution 2-D phase image with the synthetic aperture algorithm as well as the emulated DIC image. The 3-D morphology of the rod-shape phytoplankton and the nucleus

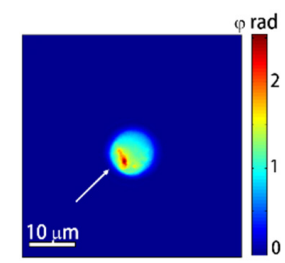

(a)

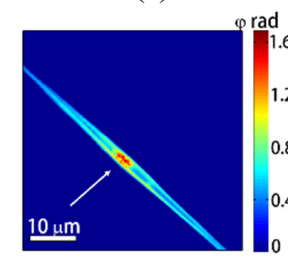

(d)

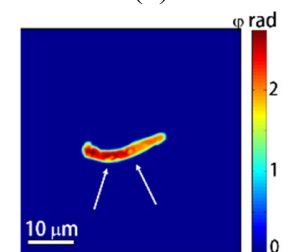

(g)

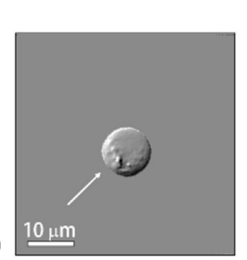

(b)

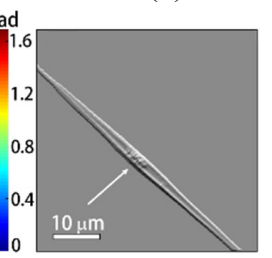

(e)

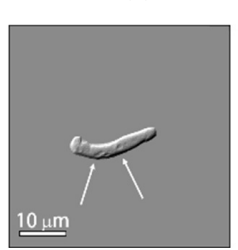

(h)

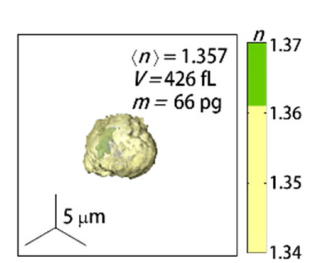

(c)

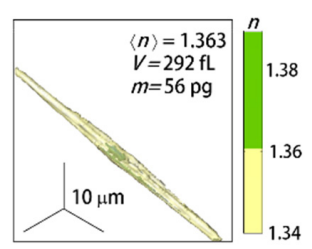

(f)

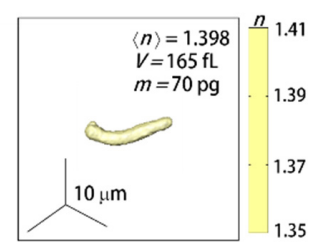

(i)
FIG. 4. Representative QPI results of individual spherical phytoplankton (a)-(c), rod-shaped phytoplankton (d)-(f), and non-spherical phytoplankton (g)-(i). (a), (d), and (g) 2-D high-resolution phase images obtained with the synthetic aperture algorithm. Units for colorbars are radians. (b), (e), and (h) emulated DIC images corresponding to (a), (d), and (g). (c), (f), and (i) 3-D RI maps of corresponding phytoplankton. The calculated mean RI $\langle n\rangle$, total volume $V$, and dry mass $M$ of individual phytoplankton, are specified. are also seen in the retrieved 3-D RI maps. The representative non-spherical phytoplankton (Figs. 4(g)-(i)) is thought to be Navicula sp. based on its shape and size seen in the measured quantitative phase images. Compared to the spherical and the rod-shaped phytoplankton, the non-spherical phytoplankton has high RI values $(\langle n\rangle=1.398)$ for its cytoplasm, indicating the high density of biomolecules in cytoplasm. Furthermore, the large nucleus shown in the spherical and the rod-shaped phytoplankton is not found in the rod-shaped phytoplankton. Yet, it contains several small internal structures with low RI values dispersed in the cytoplasm, as indicated with the arrows in Figs. $4(\mathrm{~g})-(\mathrm{h})$.

\subsection{Quantitative Phytoplankton Parameters: Volume, Dry Mass, and Density}

In order to demonstrate quantitative analysis capabilities of quantitative phase imaging of phytoplankton, we retrieved morphological and biochemical properties of individual phytoplankton. The reconstructed 3-D RI maps and 2-D quantitative phase images using the synthetic aperture algorithm allow the quantitative analysis of phytoplankton, which provides various parameters including the volume, dry mass, and cellular density of individual phytoplankton, simultaneously. From the measured volume and dry mass, we also can calculate the density of each phytoplankton. These analyses were performed to 4 spherical and 11 rod-shaped phytoplankton and the results are represented in Fig. 5. We did not conduct statistical analysis for the non-spherical phytoplankton due to their large diversity of sizes and shapes.

The volumes of individual phytoplankton are obtained from the measured 3-D RI maps. The accumulated number of voxels in a 3-D RI map of individual phytoplankton having the RI value greater than that of surrounding media was translated to the total volume considering the volume of a unit voxel. Figure 5(a) presents the volume distribution of the spherical and rod-shaped phytoplankton group. The mean values of cellular volumes of individual phytoplankton are $242 \pm 130 \mathrm{fL}(n=4)$ and $239 \pm 108 \mathrm{fL}(n=11)$, for the spherical and the rod-shaped phytoplankton, respectively. The error term means a standard deviation of the parameter. The dry masses of non-aqueous biomolecules [35-37] in individual phytoplankton are determined from the measured high-resolution 2-D quantitative phase images using the synthetic aperture algorithm. The integration of optical path length values over an area of individual phytoplankton is linearly proportional to the total dry mass in the phytoplankton via a proportional constant called RI increment [37], because the RI of biomaterials such as cells and tissues is linearly proportional to the concentration of nonaqueous biomolecules. Because the values of RI increment do not significantly vary among different species of phytoplankton, we used the report value of the RI increment $0.18 \mathrm{~mL} / \mathrm{g}$ [38] for the analysis. The result is shown in Fig. 5(b). The mean values of the determined dry masses are $36 \pm 19 \mathrm{pg}$ and $36 \pm 27 \mathrm{pg}$, for the spherical and the 


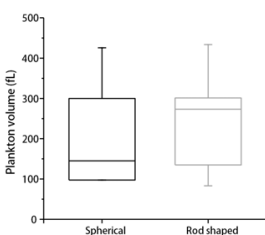

(a)

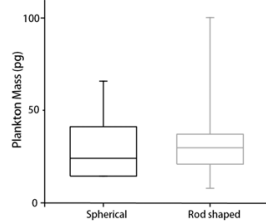

(b)

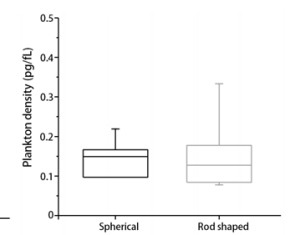

(c)
FIG. 5. Statistical analysis of quantitative parameters in QPI results of phytoplankton in spherical and rod shapes. A box-and-whisker plot of (a) volume, (b) dry mass, and (c) cytoplasmic density of spherical and rod-shaped phytoplankton are represented. Boxes, median values with upper and lower quartiles; whiskers, range. The spherical and rod-shaped phytoplankton groups contain 4 and 11 samples, respectively.

rod-shaped phytoplankton, respectively. Then, the dry mass density of individual phytoplankton is calculated by dividing the total dry mass of phytoplankton by its volume. The result is shown in Fig. 5(c). The mean values of the dry mass densities are $0.16 \pm 0.04 \mathrm{pg} / \mathrm{fL}$ and $0.15 \pm 0.08 \mathrm{pg} / \mathrm{fL}$ for the spherical and the rod-shaped phytoplankton, respectively.

\section{DISCUSSION AND CONCLUSION}

In this paper, we propose and experimentally demonstrate that the QPI techniques provide unique advantages for the study of individual phytoplankton. High-resolution 3-D RI maps, 2-D synthetic aperture images, and emulated DIC images of live phytoplankton are quantitatively and precisely measured. In particular, we show that from the measured quantitative phase images, the structure and biochemical parameters, including phytoplankton volume, total dry mass, and cytoplasmic density are simultaneously retrieved. Furthermore, internal structures of phytoplankton are clearly visualized without using exogenous labeling agents.

QPI techniques, with their capacity for 3D RI imaging, their potential for generating contrast by intrinsic optical property and their quantitative and non-invasive nature, would be a welcome complement to the existing imaging tools for the study of diverse aspects in phytoplankton biology and biophysics; (1) QPI techniques could be useful for investigating precise morphological and compositional alterations in phytoplankton under diverse environmental conditions, because the RI distributions can serve as an intrinsic optical indicator reflecting the structural and biochemical information. (2) Even though the particular focus of this study is on static 3-D images of phytoplankton, dynamics of 3-D RI tomograms of individual phytoplankton can allow to measure intracellular dynamics of subcellular organelles [39-41] or dynamics fluctuation in cell membrane [42-47] which can provide abundant information about pathophysiology of phytoplankton. (3) In addition, recent advances in QPI techniques can also be further employed to investigate phytoplankton research including super-resolution imaging
[48], Fourier transform light scattering technique [49-53], real-time visualization of 3-D RI maps [54], multi-spectral QPI [55-59], and polarization-sensitive QPI [60, 61]. (4) Furthermore, QPI techniques can be accessible to an existing microscope by attaching the recently developed QPI unit $[62,63]$, which will further extend the applicability of QPI techniques for the study of phytoplankton.

With QPI techniques, many interesting open questions in phytoplankton biology are now accessible to direct experimental study. Furthermore, the present approach can also be extended for the study of general plankton [17, 64, 65] or zoospores of alga [66]. This new approach has many potential applications. For example, when combined with underwater electronics, 3-D RI imaging of live phytoplankton could be of particular interest to marine microbiologists trying to study biological oceanography [67] and plankton ecology [68]. In addition, 3-D RI tomogram of individual phytoplankton can also be utilized for automated plankton identification [69] or the study of fluid dynamics of plankton $[62,70,71]$.

\section{ACKNOWLEDGMENT}

This work was supported by KAIST, the Korean Ministry of Education, Science and Technology and National Research Foundation (NRF) of Korea (2012R1A1A1009082, 2012M3C1A1-048860, 2013R1A1A3011886, 2013M3C1A3063046, 2013K1A3A1A09076135), APCTP, and KUSTAR-KAIST project. K.K. is supported by Global Ph.D. Fellowship from NRF.

\section{REFERENCES}

1. P. J. Lopez, J. Descles, A. E. Allen, and C. Bowler, "Prospects in diatom research," Current Opinion in Biotechnology 16, 180-186 (2005).

2. D. J. Stephens and V. J. Allan, "Light microscopy techniques for live cell imaging," Science 300, 82-86 (2003).

3. F. Zernike, "Phase contrast, a new method for the microscopic observation of transparent objects," Physica 9, 686-698 (1942).

4. G. Nomarski, "Differential microinterferometer with polarized waves," J. Phys. Radium 16, 9S-11S (1955).

5. C. Sheppard, D. Shotton, and C. Sheppard, Confocal Laser Scanning Microscopy (BIOS Scientific Publishers Oxford, 1997).

6. B. N. Giepmans, S. R. Adams, M. H. Ellisman, and R. Y. Tsien, "The fluorescent toolbox for assessing protein location and function," Science 312, 217-224 (2006).

7. G. Popescu, Quantitative Phase Imaging of Cells and Tissues (McGraw-Hill Professional, 2011).

8. K. Lee, K. Kim, J. Jung, J. H. Heo, S. Cho, S. Lee, G. Chang, Y. J. Jo, H. Park, and Y. K. Park, "Quantitative phase imaging techniques for the study of cell pathophysiology: From principles to applications," Sensors 13, 4170-4191 (2013).

9. M. K. Kim, Digital Holographic Microscopy: Principles, Techniques, and Applications (Springer, 2011). 
10. M.-K. Kim, "Applications of digital holography in biomedical microscopy," J. Opt. Soc. Korea 14, 77-89 (2010).

11. F. Yi, C.-G. Lee, and I.-K. Moon, "Statistical analysis of 3D volume of red blood cells with different shapes via digital holographic microscopy," J. Opt. Soc. Korea 16, 115-120 (2012).

12. J. Jung, K. Kim, H. Yu, K. Lee, S. Lee, S. Nahm, H. Park, and Y. Park, "Biomedical applications of holographic microspectroscopy [Invited]," Appl. Opt. 53, G111-G122 (2014).

13. Y. Kim, H. Shim, K. Kim, H. Park, J. H. Heo, J. Yoon, C. Choi, S. Jang, and Y. Park, "Common-path diffraction optical tomography for investigation of three-dimensional structures and dynamics of biological cells," Opt. Express 22, 10398-10407 (2014).

14. E. Malkiel, O. Alquaddoomi, and J. Katz, "Measurements of plankton distribution in the ocean using submersible holography," Measurement Science and Technology 10, 1142 (1999).

15. G. L. Stewart, J. R. Beers, and C. Knox, "Application of holographic techniques to the study of marine plankton in the field and in the laboratory," in Proc. Annual Technical Symposium (International Society for Optics and Photonics, 1974), pp. 183-188.

16. L. Heflinger, G. Stewart, and C. Booth, "Holographic motion pictures of microscopic plankton," Appl. Opt. 17, 951-954 (1978).

17. J. Garcia-Sucerquia, W. Xu, S. K. Jericho, P. Klages, M. H. Jericho, and H. J. Kreuzer, "Digital in-line holographic microscopy," Appl. Opt. 45, 836-850 (2006).

18. J. Garcia-Sucerquia, W. Xu, S. Jericho, M. Jericho, I. Tamblyn, and H. Kreuzer, "Digital in-line holography: 4-D imaging and tracking of micro-structures and organisms in microfluidics and biology," in Proc. ICO20: Biomedical Optics (International Society for Optics and Photonics, 2006), pp. 602613-602613-602619.

19. J. Sheng, E. Malkiel, J. Katz, J. Adolf, R. Belas, and A. R. Place, "Digital holographic microscopy reveals prey-induced changes in swimming behavior of predatory dinoflagellates," Proceedings of the National Academy of Sciences 104, 17512-17517 (2007).

20. N. I. Lewis, W. Xu, S. K. Jericho, H. J. Kreuzer, M. H. Jericho, and A. D. Cembella, "Swimming speed of three species of Alexandrium (Dinophyceae) as determined by digital in-line holography," Phycologia 45, 61-70 (2006).

21. J.-P. Hermand, J. Randall, F. Dubois, P. Queeckers, C. Yourassowsky, F. Roubaud, J. Grelet, G. Roudaut, A. Sarre, and P. Brehmer, "In-situ holography microscopy of plankton and particles over the continental shelf of Senegal," in Proc. Ocean Electronics (SYMPOL) (IEEE, 2013), pp. 1-10.

22. B. Javidi, I. Moon, S. Yeom, and E. Carapezza, "Threedimensional imaging and recognition of microorganism using single-exposure on-line (SEOL) digital holography," Opt. Express 13, $4492-4506$ (2005).

23. M. Debailleul, B. Simon, V. Georges, O. Haeberlé, and V. Lauer, "Holographic microscopy and diffractive microtomography of transparent samples," Measurement Science and Technology 19, 074009 (2008).

24. V. Lauer, "New approach to optical diffraction tomography yielding a vector equation of diffraction tomography and a novel tomographic microscope," Journal of Microscopy 205,
165-176 (2002).

25. Y. Sung, W. Choi, C. Fang-Yen, K. Badizadegan, R. R. Dasari, and M. S. Feld, "Optical diffraction tomography for high resolution live cell imaging," Opt. Express 17, 266-277 (2009).

26. K. Kim, H.-O. Yoon, M. Diez-Silva, M. Dao, R. Dasari, and Y.-K. Park, "High-resolution three-dimensional imaging of red blood cells parasitized by Plasmodium falciparum and in situ hemozoin crystals using optical diffraction tomography," J. Biomed. Opt. 19, 011005-011012 (2014).

27. S. Alexandrov, T. Hillman, T. Gutzler, and D. Sampson, "Synthetic aperture Fourier holographic optical microscopy," Phys. Rev. Lett. 97, 168102 (2006).

28. C. Fang-Yen, W. Choi, Y. J. Sung, C. J. Holbrow, R. R. Dasari, and M. S. Feld, "Video-rate tomographic phase microscopy," J. Biomed. Opt. 16, 011005 (2011).

29. S. K. Debnath, and Y. Park, "Real-time quantitative phase imaging with a spatial phase-shifting algorithm," Opt. Lett. 36, 4677-4679 (2011).

30. V. Mico, Z. Zalevsky, P. García-Martínez, and J. García, "Synthetic aperture superresolution with multiple off-axis holograms," JOSA A 23, 3162-3170 (2006).

31. T. R. Hillman, T. Gutzler, S. A. Alexandrov, and D. D. Sampson, "High-resolution, wide-field object reconstruction with synthetic aperture Fourier holographic optical microscopy," Opt. Express 17, 7873-7892 (2009).

32. N. Lue, W. Choi, G. Popescu, R. R. Dasari, K. Badizadegan, and M. S. Feld, "Quantitative phase imaging of live cells using fast Fourier phase microscopy," Appl. Opt. 46, 1836 (2007).

33. E. Wolf, "Three-dimensional structure determination of semi-transparent objects from holographic data," Opt. Commun. 1, 4 (1969).

34. K. Tam and V. Perez-Mendez, "Tomographical imaging with limited-angle input," JOSA 71, 582-592 (1981).

35. R. Barer, "Determination of dry mass, thickness, solid and water concentration in living cells," Nature 172, 1097-1098 (1953).

36. T. A. Zangle and M. A. Teitell, "Live-cell mass profiling: An emerging approach in quantitative biophysics," Nature methods 11, 1221-1228 (2014).

37. G. Popescu, Y. Park, N. Lue, C. Best-Popescu, L. Deflores, R. R. Dasari, M. S. Feld, and K. Badizadegan, "Optical imaging of cell mass and growth dynamics," Am. J. Physiol. Cell Physiol. 295, C538-544 (2008).

38. D. Stramski, "Refractive index of planktonic cells as a measure of cellular carbon and chlorophyll a content," Deep Sea Research Part I: Oceanographic Research Papers 46, 335-351 (1999).

39. Z. Wang, L. Millet, V. Chan, H. Ding, M. U. Gillette, R. Bashir, and G. Popescu, "Label-free intracellular transport measured by spatial light interference microscopy," J. Biomed. Opt. 16, 026019 (2011).

40. M. Diez-Silva, Y. Park, S. Huang, H. Bow, O. MercereauPuijalon, G. Deplaine, C. Lavazec, S. Perrot, S. Bonnefoy, M. S. Feld, J. Han, M. Dao, and S. Suresh, "Pf155/RESA protein influences the dynamic microcirculatory behavior of ring-stage Plasmodium falciparum infected red blood cells," Sci. Rep. 2, 614 (2012).

41. R. Chandramohanadas, Y. Park, L. Lui, A. Li, D. Quinn, 
K. Liew, M. Diez-Silva, Y. Sung, M. Dao, C. T. Lim, P. R. Preiser, and S. Suresh, "Biophysics of malarial parasite exit from infected erythrocytes," PLoS One 6, e20869 (2011).

42. Y. Park, C. A. Best, T. Auth, N. S. Gov, S. A. Safran, G. Popescu, S. Suresh, and M. S. Feld, "Metabolic remodeling of the human red blood cell membrane," Proceedings of the National Academy of Sciences of the United States of America 107, 1289-1294 (2010).

43. Y. Park, C. A. Best, K. Badizadegan, R. R. Dasari, M. S. Feld, T. Kuriabova, M. L. Henle, A. J. Levine, and G. Popescu, "Measurement of red blood cell mechanics during morphological changes," Proceedings of the National Academy of Sciences of the United States of America 107, 6731-6736 (2010).

44. Y. Park, M. Diez-Silva, G. Popescu, G. Lykotrafitis, W. Choi, M. S. Feld, and S. Suresh, "Refractive index maps and membrane dynamics of human red blood cells parasitized by Plasmodium falciparum," Proc. Natl. Acad. Sci. USA 105, 13730-13735 (2008).

45. G. Popescu, Y. Park, W. Choi, R. Dasari, M. Feld, and K. Badizadegan, "Imaging red blood cell dynamics by quantitative phase microscopy," Blood Cells Mol. Dis. 41, 10-16 (2008).

46. Y. Kim, H. Shim, K. H. Kim, H. J. Park, S. S. Jang, and Y. K. Park, "Profiling individual human red blood cells using common-path diffraction optical tomography," Scientific Reports 4 (2014).

47. S. Oh, C. Fang-Yen, W. Choi, Z. Yaqoob, D. Fu, Y. K. Park, R. R. Dassari, and M. S. Feld, "Label-free imaging of membrane potential using membrane electromotility," Biophys. J. 103, 11-18 (2012).

48. Y. Cotte, F. Toy, P. Jourdain, N. Pavillon, D. Boss, P. Magistretti, P. Marquet, and C. Depeursinge, "Marker-free phase nanoscopy," Nature Photonics 7, 113-117 (2013).

49. H. Ding, Z. Wang, F. Nguyen, S. A. Boppart, and G. Popescu, "Fourier transform light scattering of inhomogeneous and dynamic structures," Phys. Rev. Lett. 101, 238102 (2008).

50. K. Lee, H.-D. Kim, K. Kim, Y. Kim, T. R. Hillman, B. Min, and Y. Park, "Synthetic Fourier transform light scattering," Opt. Express 21, 22453-22463 (2013).

51. Y. Jo, J. Jung, J. W. Lee, D. Shin, H. Park, K. T. Nam, J.-H. Park, and Y. Park, "Angle-resolved light scattering of individual rod-shaped bacteria based on Fourier transform light scattering," Scientific Reports 4 (2014).

52. K. Kim and Y. Park, "Fourier transform light scattering angular spectroscopy using digital inline holography," Opt. Lett. 37, 4161-4163 (2012).

53. Y. Kim, J. M. Higgins, R. R. Dasari, S. Suresh, and Y. K. Park, "Anisotropic light scattering of individual sickle red blood cells," J. Biomed. Opt. 17, 040501-040501 (2012).

54. K. Kim, K. S. Kim, H. Park, J. C. Ye, and Y. Park, "Real-time visualization of 3-D dynamic microscopic objects using optical diffraction tomography," Opt. Express 21, 32269-32278 (2013).

55. Y. Park, T. Yamauchi, W. Choi, R. Dasari, and M. S. Feld, "Spectroscopic phase microscopy for quantifying hemoglobin concentrations in intact red blood cells," Opt. Lett. 34, 3668-3670 (2009).

56. Y. Jang, J. Jang, and Y. Park, "Dynamic spectroscopic phase microscopy for quantifying hemoglobin concentration and dynamic membrane fluctuation in red blood cells," Opt. Express 20 (2012).

57. J. Jung, J. Jang, and Y. Park, "Spectro-refractometry of individual microscopic objects using swept-source quantitative phase imaging," Anal. Chem. 85, 10519-10525 (2013).

58. J. Jung and Y. Park, "Spectro-angular light scattering measurements of individual microscopic objects," Opt. Express 22, 4108-4114 (2014).

59. J. Jung, K. Kim, H. Yu, K. Lee, S. Lee, S. Nahm, H. Park, and Y. Park, "Biomedical applications of holographic micro-spectroscopy," Appl. Opt. 53, G111-G122 (2014).

60. Z. Wang, L. J. Millet, M. U. Gillette, and G. Popescu, "Jones phase microscopy of transparent and anisotropic samples," Opt. Lett. 33, 1270-1272 (2008).

61. Y. Kim, J. Jeong, J. Jang, M. W. Kim, and Y. Park, "Polarization holographic microscopy for extracting spatiotemporally resolved Jones matrix," Opt. Express 20 (2012).

62. S. J. Lee, H. J. Byeon, and K. W. Seo, "Inertial migration of spherical elastic phytoplankton in pipe flow," Experiments in Fluids 55, 1-9 (2014).

63. K. Kim, Z. Yaqoob, K. Lee, J. W. Kang, Y. Choi, P. Hosseini, P. T. So, and Y. Park, "Diffraction optical tomography using quantitative phase imaging unit," Opt. Lett. (to be published).

64. K. Kim, Z. Yaqoob, K. Lee, J. W. Kang, Y. Choi, P. Hosseini, P. T. So, and Y. Park, "Diffraction optical tomography using quantitative phase imaging unit," Opt. Lett. 39, 69356938 (2014).

65. H. Sun, P. Benzie, N. Burns, D. Hendry, M. Player, and J. Watson, "Underwater digital holography for studies of marine plankton," Philosophical Transactions of the Royal Society A: Mathematical, Physical and Engineering Sciences 366, 1789-1806 (2008).

66. M. Heydt, A. Rosenhahn, M. Grunze, M. Pettitt, M. Callow, and J. Callow, "Digital in-line holography as a three-dimensional tool to study motile marine organisms during their exploration of surfaces," The Journal of Adhesion 83, 417-430 (2007).

67. P. H. Wiebe and M. C. Benfield, "From the Hensen net toward four-dimensional biological oceanography," Progress in Oceanography 56, 7-136 (2003).

68. H.-U. Dahms and J.-S. Hwang, "Perspectives of underwater optics in biological oceanography and plankton ecology studies," Journal of Marine Science and Technology 18, 112-121 (2010).

69. M. C. Benfield, P. Grosjean, P. F. Culverhouse, X. Irigoien, M. E. Sieracki, A. Lopez-Urrutia, H. G. Dam, Q. Hu, C. S. Davis, A. Hansen, C. H. Pilskaln, E. M. Riseman, H. Schultz, P. E. Utgoff, and G. Gorsky, "RAPID Research on automated plankton identification," Oceanography 20, 172-187 (2007).

70. W. M. Durham, E. Climent, M. Barry, F. De Lillo, G. Boffetta, M. Cencini, and R. Stocker, "Turbulence drives microscale patches of motile phytoplankton," Nature Communications 4, 2148 (2013).

71. M. H. Sohn, S. Lim, K. W. Seo, and S. J. Lee, "Effect of ambient medium viscosity on the motility and flagella motion of Prorocentrum minimum (Dinophyceae)," Journal of Plankton Research 35, 1294-1304 (2013). 